\title{
Neutrinos from the pulsar wind nebulae
}

\author{
W. Bednarek ${ }^{\star}$ \\ Department of Experimental Physics, University of Łódź, ul. Pomorska 149/153, 90-236 Łódź, Poland
}

Received 13 February 2003 / Accepted 20 May 2003

\begin{abstract}
In a recent paper we calculated the $\gamma$-ray spectra from pulsar wind nebulae (PWNe), assuming that a significant amount of the pulsar rotational energy is converted into relativistic nuclei. These nuclei accelerate leptons which are responsible for most of the observed electromagnetic emission from PWNe. A small part of nuclei also interact with the matter of the supernova producing $\gamma$-rays, which can also contribute to the observed spectra of young nebulae. Here we calculate the spectra of neutrinos from the interaction of nuclei inside the nebula and the expected neutrino event rates in the $1 \mathrm{~km}^{2}$ neutrino detector from the Crab Nebula (PSR 0531+21), the Vela SNR (PSR 0833-45), G 343.1-2.3 (PSR 1706-44), MSH15-52 (PSR 1509-58), 3C 58 (PSR J0205+6449), and CTB80 (PSR 1951+32). It is shown that only the Crab Nebula can produce neutrino event rate above the sensitivity limit of the $1 \mathrm{~km}^{2}$ neutrino detector, provided that nuclei take most of the rotational energy lost by the pulsar. The neutrino event rate expected from the Vela SNR is comparable to that of the Crab Nebula but these neutrinos are less energetic and are emitted from a much larger region on the sky. Therefore it may be difficult to subtract the Vela SNR signal from the higher background of atmospheric neutrinos.
\end{abstract}

Key words. ISM: supernova remnants - stars: pulsars: general - radiation mechanisms: non-thermal

\section{Introduction}

The possibility that hadronic processes can contribute to the observed $\gamma$-ray emission from the PWNe has been considered in recent years by e.g. Cheng et al. (1990) and Aharonian \& Atoyan (1996). Following these works and earlier results (e.g. Berezinsky \& Prilutsky 1978), we discussed a model for the production of radiation in hadronic processes with the application to the very young PWNe (Protheroe et al. 1998; Beall $\&$ Bednarek 2002). In these papers, the $\gamma$-ray and/or neutrino fluxes were estimated from the interaction of nuclei, injected by the pulsar, with the matter and soft radiation of the supernova remnant during its early phase of development, i.e. within the first few years. A general model like this has recently become very popular, finding its application in the estimation of neutrino fluxes from the $\gamma$-ray bursts in terms of the supranova model (e.g. Guetta \& Granot 2002; Razzaque et al. 2002; Dermer \& Atoyan 2003), which postulates the formation of a very fast pulsar during supernova explosion (Vietri \& Stella 1998). In another paper the contribution of nuclei from the pulsar to the $\gamma$-ray (and neutrino) spectrum was also calculated for the best known example of an older supernova remnant, i.e. the Crab Nebula (Bednarek \& Protheroe 1997). Recently, the neutrino flux from the Crab Nebula has been independently estimated by Amato et al. (2003). The authors predict a few to several neutrino events in a $1 \mathrm{~km}^{2}$ detector per year from this source.

\footnotetext{
* e-mail: bednar@fizwe4.fic.uni.lodz.pl
}

Recently we have considered a more general model for the hadronic and leptonic processes inside the PWNe, which are based on the proposition that a significant part of the pulsar rotational energy is taken up by relativistic nuclei (Arons and collaborators, e.g. Arons 1998). Our purpose was to calculate the $\gamma$-ray spectra from well-known PWNe. From the comparison of the calculations with the observations of these $\gamma$-ray nebulae, we derived some free parametrs of the considered model in order to predict the $\gamma$-ray and neutrino fluxes from other nebulae, which may become potential targets for the next generation of $\gamma$-ray and neutrino telescopes. The calculations of the $\gamma$ ray spectra from leptonic and hadronic processes were reported in the accompanying paper by Bednarek \& Bartosik (2003, BB03). The neutrino spectra are calculated in the present paper. Most of the earlier calculations of the $\gamma$-ray fluxes from the PWNe, which refer mainly to the Crab Nebula (e.g. De Jager \& Harding 1992; Aharonian \& Atoyan 1995; De Jager et al. 1996; Hillas et al. 1998), were done based only on the leptonic origin for this emission. The calculations of the $\gamma$-ray fluxes from other nebulae are available only for a few objects, e.g. nebulae around PSR 1706-44 (Aharonian et al. 1997), PSR 1509-58 (Du Plessis et al. 1995), Vela pulsar (De Jager et al. 1996).

The neutrino fluxes from a few PWNe have recently been re-scaled from the observed $\mathrm{TeV} \gamma$-ray fluxes by Guetta \& Amato (2002), based on the simple assumption that in all these nebulae the $\gamma$-rays above $2 \mathrm{TeV}$ are produced in the decay of $\pi^{\circ}$, which originate in hadronic processes. This assumption is in contradiction to the popular opinion on the relative importance 
of the leptonic and hadronic processes in PWNe and also with the results presented in this paper.

\section{The model for high energy processes in the pulsar wind nebula}

The details of the model for high energy processes in the PWNe are described in our first paper (BB03). For consistency we repeat here its main features. The evolution of the supernova remnant, containing an energetic pulsar, is described following the main points of the picture considered soon after the discovery of pulsars by Ostriker \& Gunn (1971) and Rees \& Gunn (1974). Let us assume that when the explosion occurs, the expansion velocity of the nebula at its inner radius is $V_{0, \mathrm{SN}}$ and its initial mass is $M_{0, \mathrm{SN}}$. However, this expansion velocity can increase due to the additional supply of energy to the nebula by the pulsar and can also decrease due to the accumulation of the surrounding matter. We take these processes into account when determining the radius of the nebula at the specific time, $t$, by using the energy conservation,

$\frac{M_{\mathrm{SN}}(t) V_{\mathrm{SN}}^{2}(t)}{2}=\frac{M_{0, \mathrm{SN}} V_{0, \mathrm{SN}}^{2}}{2}+\int_{0}^{t} L_{\mathrm{em}}\left(t^{\prime}\right) \mathrm{d} t^{\prime}$,

where

$L_{\mathrm{em}}(t)=B_{\mathrm{s}}^{2} R_{\mathrm{s}}^{6} \Omega^{4} / 6 c^{3} \approx 1.3 \times 10^{45} B_{12}^{2} P_{\mathrm{ms}}^{-4} \operatorname{erg~s}^{-1}$,

is the pulsar energy loss on emission of dipole electromagnetic radiation, $\Omega=2 \pi / P$, and the period of the pulsar $P=$ $10^{-3} P_{\mathrm{ms}} \mathrm{s}$ changes with time according to

$P_{\mathrm{ms}}^{2}(t)=P_{0, \mathrm{~ms}}^{2}+2 \times 10^{-9} t B_{12}^{2}$,

where $P_{0, \mathrm{~ms}}$ is the initial period of the pulsar and $B=10^{12} B_{12} \mathrm{G}$ the strength of its surface magnetic field. The expanding nebula increases the mass from the surrounding medium according to

$M_{\mathrm{SN}}(t)=M_{0, \mathrm{SN}}+\frac{4}{3} \pi \rho_{\mathrm{sur}} R_{\mathrm{Neb}}^{3}(t)$,

where $\rho_{\text {sur }}$ is the density of the surrounding medium and $R_{\mathrm{Neb}}$ is the outer radius of expanding envelope at the time, $t$, which depends on the expansion history of the nebula,

$R_{\mathrm{Neb}}=\int_{0}^{t} V_{\mathrm{SN}}\left(t^{\prime}\right) \mathrm{d} t^{\prime}$.

The expansion velocity of the nebula, $V_{\mathrm{SN}}(t)$, and its density of matter, $\rho_{\mathrm{Neb}}=3 M_{\mathrm{SN}}(t) / 4 \pi R_{\mathrm{Neb}}^{3}(t)$, at time $t$ have been found by solving the above set of Eqs. (1)-(5) numerically.

The pulsar loses energy in the form of relativistic wind which extends up to the distance $R_{\mathrm{sh}}$. At this distance, the pressure of the wind is balanced by the pressure of the expanding nebula. We estimate the location of this shock at time $t$ by comparing the wind energy flux, determined by $L_{\mathrm{em}}$ (Eq. (2)), with the pressure of the outer nebula, determined by the supply of energy to the nebula by the pulsar over the whole of its lifetime (Rees \& Gunn 1974),

$$
\frac{L_{\mathrm{em}}(t)}{4 \pi R_{\mathrm{sh}}^{2} c} \approx \frac{\int_{0}^{t} L_{\mathrm{em}}\left(t^{\prime}\right) \mathrm{d} t^{\prime}}{\frac{4}{3} \pi R_{\mathrm{Neb}}^{3}}
$$

The assumption that the pulsar wind ram pressure is balanced by the total pressure inside the nebula is different from that made in the previous paper (BB03) in which the wind pressure is balanced only by the magnetic field pressure. In fact this modification changes the location of the pulsar wind shock for very young nebulae but does not influence the calculated neutrino fluxes from them since the escape conditions of heavy nuclei from the nebula do not change significantly.

Knowing how the magnetic field depends on the distance from the pulsar in the pulsar wind zone, we can estimate the strength of the magnetic field in the shock region from

$B_{\mathrm{sh}}=\sqrt{\sigma} B_{\mathrm{pul}}\left(\frac{R_{\mathrm{pul}}}{R_{\mathrm{lc}}}\right)^{3} \frac{R_{\mathrm{lc}}}{R_{\mathrm{sh}}}$,

where $\sigma$ is the ratio of the magnetic energy flux to the particle energy flux from the pulsar at the location of the pulsar wind shock, $R_{\text {pul }}$ and $B_{\text {pul }}$ are the radius and the surface magnetic field of the pulsar, respectively. The evolution of $\sigma$ with the parameters of the pulsar is found by interpolating between the values estimated for the Crab pulsar, $\sim 0.003$, and for the Vela pulsar, 1 (see Eq. (16) and below in Bednarek \& Protheroe 2002 for details).

It is likely that a significant part of the rotational energy of the pulsar, transfered through the shock radius $R_{\mathrm{sh}}$, is in the form of relativistic heavy nuclei. In fact, such an assumption can explain morphological features of the Crab Nebula and also the appearance of extremely energetic leptons inside the nebula accelerated as a result of the resonant scattering of positrons and electrons by heavy nuclei (Hoshino et al. 1992; Gallant $\&$ Arons 1994). From the normalization to the observations of the Crab pulsar, Arons and collaborators (see e.g. Arons 1998) postulate that the Lorenz factors of iron nuclei in the pulsar's wind should be

$\gamma_{1} \approx 0.3 Z e \Phi_{\text {open }} / m_{\mathrm{i}} c^{2}$,

where $m_{\mathrm{i}}$ and $Z e$ are the mass and charge of the iron nuclei, $c$ is the velocity of light, and $\Phi_{\text {open }}=\sqrt{E_{\mathrm{em}} / c}$ is the total electric potential drop across the open magnetosphere. Equation (8) postulates that a pulsar with a specific period and a surface magnetic field accelerates nuclei monoenergetically. As assumed in the Gallant \& Arons (1994) model for the Crab Nebula, the nuclei take a significant part, $\chi$, of the total rotational energy lost by the pulsar. The calculations of neutrino rates from the PWMe, presented below, are done for the value of $\chi=0.8$. In the next section we integrate the injection spectra of nuclei over the activity period of the pulsar in order to obtain the equilibrium spectrum of the nuclei inside the nebula at a specific time after pulsar formation.

\section{Production of neutrinos by nuclei}

The nuclei injected into the nebula suffer adiabatic energy losses due to the expansion of the nebula and rare collisions with the matter of the nebula. The most energetic nuclei can diffuse out of the nebula. We include all these energy loss processes for the nuclei (adiabatic losses, fragmentation, escape) following Bednarek \& Protheroe (2002), and BB03, to calculate the equilibrium spectra of different types of nuclei inside 


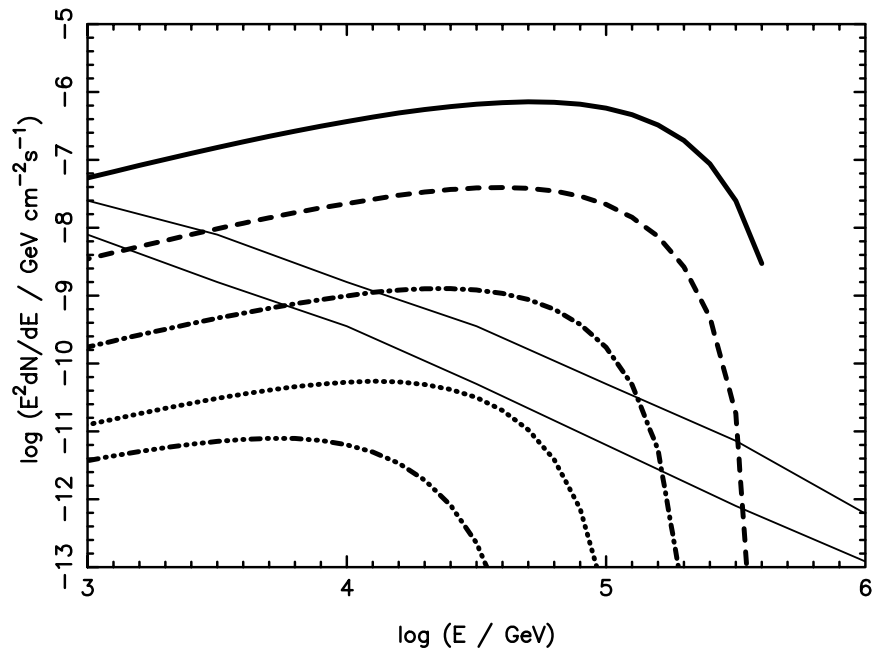

Fig. 1. The muon neutrino and antineutrino spectra from the pulsar wind nebula at the distance of $2 \mathrm{kpc}$ at different times after the pulsar formation: $t=10^{2}, 3 \times 10^{2}, 10^{3}, 3 \times 10^{3}$, and $10^{4} \mathrm{yrs}$. The initial period of the pulsar is $15 \mathrm{~ms}$ and the surface magnetic field $4 \times 10^{12} \mathrm{G}$. The supernova has a mass of $3 M_{\odot}$ and initial expansion velocity of $2000 \mathrm{~km} \mathrm{~s}^{-1}$. The atmospheric neutrino background within $1^{\circ}$ of the source, ANB, is marked by the thin full curves, horizontal (upper) and vertical (lower) (from Lipari 1993).

the nebula at a specific time after pulsar formation (see Sect. 4.1 and Fig. 2 in BB03 for example equilibrium spectra of nuclei inside the nebula).

The nuclei, with the equilibrium spectra, interact with the matter of the supernova remnant and produce neutrinos via decay of pions. We estimate the neutrino spectra by simple re-scaling of the $\gamma$-ray spectra calculated for these hadrons, applying the scaling brake model proposed by Wdowczyk \& Wolfendale (1987). The number of produced neutrinos is on average two times larger than the number of $\gamma$-rays (the multiplicity of charged pions with respect to the neutral pions is larger by this factor) and the average energies of neutrinos are a factor of two lower than the $\gamma$-rays. As an example, we show in Fig. 1 the spectra of muon neutrinos (and antineutrinos) produced at different times, $10^{2} \mathrm{yr}, 3 \times 10^{2} \mathrm{yr}, 10^{3} \mathrm{yr}, 3 \times 10^{3} \mathrm{yr}$, and $10^{4} \mathrm{yr}$ after the formation of the pulsar at the distance of $2 \mathrm{kpc}$. The initial parameters of the pulsar are: the period $15 \mathrm{~ms}$ and the surface magnetic field $4 \times 10^{12} \mathrm{G}$, and the initial parameters of the nebula (supernova remnant) are: the mass $3 M_{\odot}$ and the velocity $2000 \mathrm{~km} \mathrm{~s}^{-1}$. It is assumed that the supernova exploded in a medium with a typical density of $0.3 \mathrm{~cm}^{-3}$. We also show the atmospheric neutrino background (ANB) expected within $1^{\circ}$ of the source as calculated by Lipari (1993). As expected, the intensities of neutrino spectra strongly depend on the age of the nebula due to the lower densities of matter inside the nebula. The spectra also shift to lower energies due to the adiabatic energy losses of nuclei, more efficient escape of higher energy nuclei from the nebula, and lower energies of the freshly injected nuclei inside the nebula by older pulsars. In Fig. 2 we show also the expected neutrino event rate in a $1 \mathrm{~km}^{2}$ detector from pulsars with different parameters. The predicted event rates are above 1 per year if the age of the pulsar (and nebula) is shorter than $\sim 10^{3}-3 \times 10^{3}$ yrs.

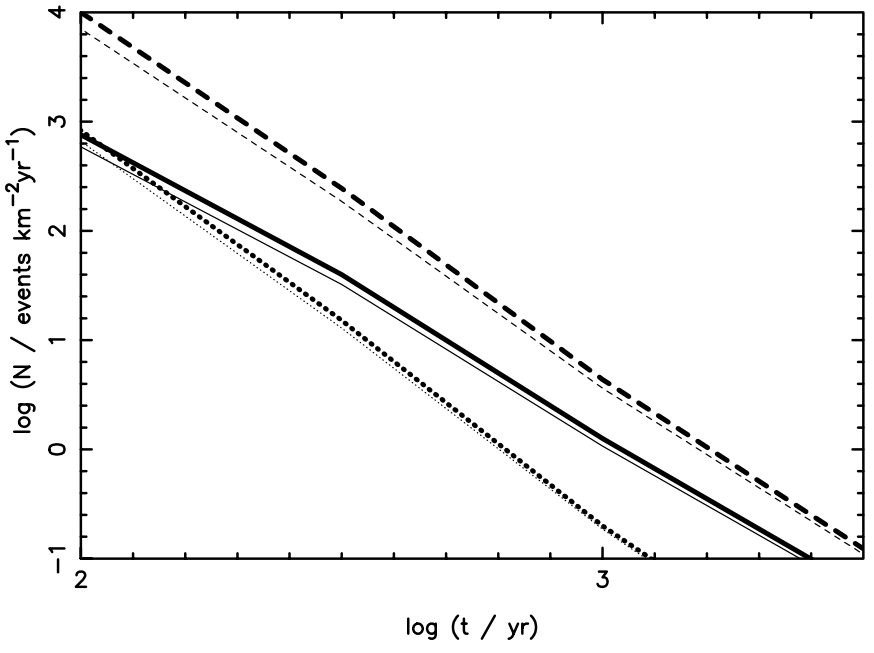

Fig. 2. The muon neutrino event rate in a $1 \mathrm{~km}^{2}$ detector from the pulsar wind nebula at the distance of $2 \mathrm{kpc}$ as a function of the time after pulsar formation for different initial parameters of the pulsar: initial period $15 \mathrm{~ms}$ and surface magnetic field $B=4 \times 10^{12} \mathrm{G}$ (full curve), $5 \mathrm{~ms}$ and $B=4 \times 10^{12} \mathrm{G}$ (dashed curve), and $15 \mathrm{~ms}$ and $B=$ $2 \times 10^{13} \mathrm{G}$ (dotted curve). The rates for the neutrino events from the nadir (absorption by the Earth) and the zenith directions are marked by the thin and thick curves, respectively.

\section{Expected neutrino rates from specific nebulae}

We calculate the expected neutrino spectra at the Earth and the neutrino event rates in a $1 \mathrm{~km}^{2}$ detector from the PWNe for which the $\gamma$-ray fluxes have been calculated in our previous paper (BB03). The initial parameters of the nebulae and the pulsars for these sources have been taken as applied in calculations of $\gamma$-ray fluxes.

To estimate the neutrino rates we applied the muon neutrino detection probabilities of the $1 \mathrm{~km}^{2}$ detector calculated by Gaisser \& Grillo (1987). The neutrinos arriving at the detector from the nadir direction can partially be absorbed by the Earth. This effect is significant for neutrinos with energies $>100 \mathrm{TeV}$. We take it into account by applying the neutrino absorption coefficients calculated by Gandhi (2000). These absorption effects are not very important in the case of most of the considered objects.

\subsection{The Crab Nebula}

The observed parameters of the Crab Nebula and the pulsar (the present radius $\sim 2-3 \mathrm{pc}$, the expansion velocity $2000 \mathrm{~km} \mathrm{~s}^{-1}$, the period $\sim 33.4 \mathrm{~ms}$ ) can be fitted by assuming that: (1) the pulsar was born with the initial period of $15 \mathrm{~ms}$, the surface magnetic field $3.8 \times 10^{12} \mathrm{G}$, as derived from the observed period and period derivative with the assumption that the pulsar loses most of its energy by the dipole electromagnetic radiation; (2) the parent Crab Nebula supernova is of $3 M_{\odot}$ (consistent with the observed density of matter inside the nebula), and expends with an initial velocity of $2000 \mathrm{~km} \mathrm{~s}^{-1}$ at a distance of 1830 pc from the Crab Nebula (Davidson \& Fesen 1985). For the applied parameters, we successfully described the observed $\gamma$-ray spectrum from the Crab Nebula (see BB03) as a 


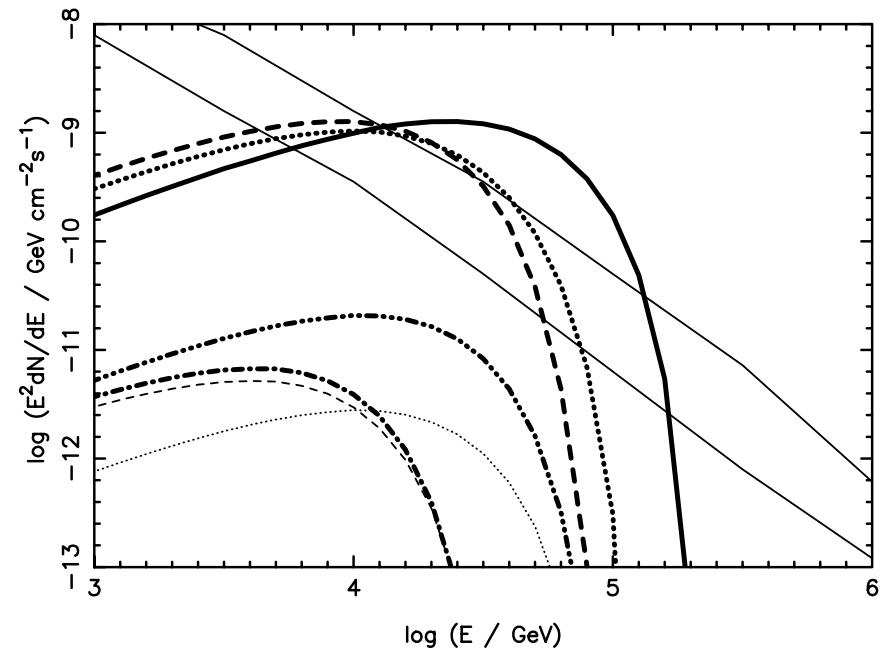

Fig. 3. Neutrino spectra $\left(v_{\mu}\right)$ expected from different nebulae: Crab Nebula - PSR 0531+21 (thick full curve), Vela Nebula - PSR 0833-45 (thick dashed), G343.1-2.3 - PSR 1706-44 (thick dot-dashed), 3C 58 - PSR J0205+6449 (thick dot-dot-dot-dashed), MSH15-52 - PSR 1509-58 (thin dotted) and with close high density medium with $300 \mathrm{~cm}^{-3}$ (thick dotted), CTB80 - PSR 1951+32 (thin dashed). In all cases, except PSR 1509-58, the parent supernova explodes in the medium with a density of $0.3 \mathrm{~cm}^{-3}$. The ANB is marked by the thin full curves.

self-consistent composition of the $\gamma$-ray spectra produced by leptons $(<10 \mathrm{TeV})$ and hadrons $(>10 \mathrm{TeV})$.

The muon neutrino and antineutrino spectrum from the Crab Nebula, calculated for the above parameters, is above the atmospheric neutrino background within $1^{\circ}$ of the source (see Fig. 3). However, the neutrino rate in the $1 \mathrm{~km}^{2}$ detector, about one event per year, is relatively low (see Table 1). This rate is similar to the neutrino rate predicted in our earlier calculations (Bednarek \& Protheroe 1997), which are based on somewhat different assumptions. In that model we assumed that neutrinos are produced in the interactions of nucleons with the matter of the expanding supernova remnant. The nucleons were dissolved from the heavy nuclei during their propagation in the inner pulsar magnetosphere.

\subsection{The Vela Supernova Remnant}

The Vela pulsar is inside the compact nonthermal nebula with a radius of $\sim 7^{\prime}$ and the extended Vela Supernova Remnant (Vela SNR) with a radius of $\sim 3.5^{\circ}$. The observed parameters of the pulsar allow us to estimate its surface magnetic field, $4.5 \times 10^{12} \mathrm{G}$, and the characteristic age of $11300 \mathrm{yrs}$. We model the Vela SNR assuming that the initial parameters of this object were similar to the Crab Nebula, i.e. an initial pulsar period of $15 \mathrm{~ms}$, the mass of the Vela SNR of $3 M_{\odot}$ and an expansion velocity of $2000 \mathrm{~km} \mathrm{~s}^{-1}$. The distance to the Vela pulsar is taken to be $\sim 300$ pc (Caraveo et al. 2001), although the older literature suggested the value of 500 pc (e.g. Cha et al. 1999). For these parameters of the pulsar and nebula, we estimate its real age to be $\sim 6000 \mathrm{yrs}$, consistent with the observed dimensions of the nebula.
The muon neutrino spectrum, calculated from the Vela SNR, is on the level of ANB within $1^{\circ}$ of the source (Fig. 3). The expected neutrino event rate is similar to the Crab Nebula neutrino event rate (Table 1), but the neutrinos from the Vela SNR are less energetic. Moreover, due to the very large size of the Vela SNR on the sky, $\sim 3.5^{\circ}$, the expected ANB for this source may be about an order of magnitude higher than marked in Fig. 3. Therefore the observation of a neutrino signal from the Vela SNR is unlikely.

\subsection{The nebula around PSR 1706-44}

The pulsar PSR 1706-44, and its nebula (G 343.1-2.3), show close similarities to the Vela pulsar and SNR. It has also been reported at $\mathrm{TeV} \gamma$-ray energies (Kifune et al. 1995; Chadwick et al. 1998). These pulsars have similar characteristic ages and present periods. However, due to the lower surface magnetic field of the PSR1706-44, equal to $3.1 \times 10^{12} \mathrm{G}$, and a longer period, $102 \mathrm{~ms}$, the real age of the pulsar should be closer to its characteristic age, $17400 \mathrm{yrs}$. As in the previous modelling, we assume that the initial period of this pulsar is also $15 \mathrm{~ms}$ and the expansion velocity of the nebula is $2000 \mathrm{~km} \mathrm{~s}^{-1}$. For these parameters of the pulsar and nebula, the consistency with the observed parameters of this object is reached for its real age, 16000 yrs. The distance to PSR 1706-44 is taken to be $1.8 \mathrm{kpc}$ (Taylor \& Cordes 1993).

The neutrino spectra calculated from PSR 1706-44 are about two orders of magnitude below the ANB (Fig. 3), and the predicted neutrino event rate in a $1 \mathrm{~km}^{2}$ detector, $4 \times$ $10^{-3}$ per year, does not allow their detection in a reasonable time. These small values are due to the significantly larger distance and higher real age of PSR 1706-44 in comparison to the Vela pulsar.

\subsection{The nebula $3 C 58$ around PSR J0205+6449}

The pulsar PSR J0205+6449 and its nebula 3C 58 is sometimes identified with the historic supernova appearing in the year 1181 (Thorsett et al. 1992). However, in such a case, using the required initial period of the pulsar of $\sim 60 \mathrm{~ms}$ and the expension velocity of the nebula of $\sim 5000 \mathrm{~km} \mathrm{~s}^{-1}$ (to fit the observed dimensions of the nebula), we were not able to describe successfully the observed electromagnetic spectrum from this source in the broad energy range (BB03).

Therefore, in order to fit the observed electromagnetic spectrum, we followed another proposition for the parameters of this pulsar and nebula: the age of 5000 yrs (Bietenholz et al. 2001), which is consistent with the characteristic age (Murray et al. 2002), initial period of $15 \mathrm{~ms}$, and expansion velocity of $1000 \mathrm{~km} \mathrm{~s}^{-1}$ (Fesen 1983). These values allow us to fit the present parameters of this system. The surface magnetic field of this pulsar is estimated to be $3.6 \times 10^{12} \mathrm{G}$ and its distance is $3.2 \mathrm{kpc}$ (Roberts et al. 1993). However, for these parameters, the neutrino spectrum from PSR J0205+6449 is about two orders of magnitude below the ANB (Fig. 3), and the predicted neutrino event rate (see Table 1) is not detectable. 
Table 1. Expected number of $v_{\mu}$ neutrinos observed by the $1 \mathrm{~km}^{2}$ detector during 1 year.

\begin{tabular}{ccccccc}
\hline \hline $\begin{array}{c}\text { Nebula } \\
(\text { pulsar })\end{array}$ & $\begin{array}{c}\text { Crab Nebula } \\
(\text { PSR0531+21) }\end{array}$ & $\begin{array}{c}\text { Vela nebula } \\
(\text { PSR0833-45) }\end{array}$ & $\begin{array}{c}\text { G 343.1-2.3 } \\
(\text { PSR1706-44) }\end{array}$ & $\begin{array}{c}\text { 3C 58 } \\
\text { (PSRJ0205+6449) }\end{array}$ & $\begin{array}{c}\text { MSH15-52 } \\
\text { (PSR1509-58) }\end{array}$ & $\begin{array}{c}\text { CTB80 } \\
(\text { PSR1951+32) }\end{array}$ \\
\hline $\begin{array}{c}\text { neutrino rate } \\
(>0.1 \mathrm{TeV})\end{array}$ & $1.3(1.1)$ & $1.15(1.05)$ & $4 \times 10^{-3}$ & 0.02 & $3 \times 10^{-3}$ & $5 \times 10^{-3}$ \\
$(1.0)$ & & $(0.03)$ & $(1.0)$ \\
\hline
\end{tabular}

\subsection{The nebula MSH15-52 around PSR 1509-58}

The pulsar PSR 1509-58, with the characteristic age of $\sim 1700 \mathrm{yr}$, and its complex nebula MSH15-52, are sometimes identified with the historic supernova SN 185 (Thorsett 1992). The distance to these objects is put in the range $4.2 \mathrm{kpc}$ (kinematics of H I, Caswell et al. 1975) to $5.9 \mathrm{kpc}$ (dispersion measure, Taylor \& Cordes 1993). We apply the value of $5.2 \mathrm{kpc}$. The pulsar has the present period of $\sim 150 \mathrm{~ms}$, and the surface magnetic field of $1.5 \times 10^{13} \mathrm{G}$. Its present parameters can be explained by assuming that the initial pulsar period was $15 \mathrm{~ms}$ and its real age is close to its characteristic age. However, in order to fit the observed dimensions of the nebula, $5^{\prime} \times 10^{\prime}$, we have to assume that the expansion velocity of the bulk matter in this nebula is $5000 \mathrm{~km} \mathrm{~s}^{-1}$, which is much higher than that applied in the modelling of other nebulae.

The neutrino spectrum calculated from this nebula is about two orders of magnitude below the ANB (Fig. 3) and the expected neutrino event rate in the $1 \mathrm{~km}^{2}$ detector is very low (Table 1). However, this source has been marginally detected by the CANGAROO telescope at $\mathrm{TeV}$ energies (Sako et al. 2000). We were not able to describe such a high level of $\gamma$-ray emission from MSH15-52 by only leptonic processes (BB03). However, the thermal, optical nebula RCW 89, containg $\mathrm{H}_{\alpha}$ line-emitting filaments with a density of $\sim 5 \times$ $10^{3} \mathrm{~cm}^{-3}$, coincides with the NW component of the remnant MSH15-52 (Seward et al. 1983). Therefore, if nuclei injected by the pulsar are captured by these high density filaments, then the reported level of $\gamma$-ray emission from this object can be explained by the $\gamma$-rays produced in interactions of nuclei with the matter having an average density of $\sim 300 \mathrm{~cm}^{-3}$. Then, the accompanying neutrino emission is comparable to the ANB (Fig. 3), and the expected neutrino event rate in a $1 \mathrm{~km}^{2} \mathrm{de}-$ tector is close to 1 event per year.

\subsection{The nebula CTB80 around PSR $1951+32$}

The pulsar PSR 1951+32 has a short period, $39.5 \mathrm{~ms}$, a high characteristic age of $1.1 \times 10^{5} \mathrm{yr}$, and a relatively weak surface magnetic field of $4.9 \times 10^{11} \mathrm{G}$. It lies inside the supernova remnant CTB80, which consists of a $10^{\prime} \times 6^{\prime}$ compact nebula and shell-like extended component with a diameter of $30^{\prime}$. The estimated dynamic age of the nebula, $9.6 \times 10^{4} \mathrm{yr}$ (for the distance $2.5 \mathrm{kpc}$ ), matches the characteristic age of the pulsar (Koo et al. 1990). Therefore, in our modelling, we assume that these objects are at a distance of $2.5 \mathrm{kpc}$ and have a real age close to the pulsar characteristic age. The observed parameters of the pulsar and nebula are consistent with the initial period of the pulsar of $15 \mathrm{~ms}$ and the expansion velocity of the nebula of $2000 \mathrm{~km} \mathrm{~s}^{-1}$. The neutrino spectrum calculated for the above parameters is also orders of magnitudes below the ANB (Fig. 3), and the predicted neutrino event rate is below the sensitivity of a $1 \mathrm{~km}^{2}$ neutrino detector (Table 1).

The nebula CTB80 is sometimes regarded as the remnant of the recent supernova that appeared in the year 1408, found in Chinese records (Strom et al. 1980; Wang \& Seward 1984). If this is the case, then the initial period of the pulsar has to be very close to the one observed and the energy lost by the pulsar is very low. Assuming the expansion velocity of the nebula equal to $1000 \mathrm{~km} \mathrm{~s}^{-1}$, to fit the observed dimension of the compact nebula, the expected neutrino event rate in this case is about an order of magnitude higher than estimated above (see the number in brackets in Table 1), but still below the sensitivity of future neutrino detectors.

\section{Conclusion}

According to the model discussed here for the high energy processes in PWNe, in which most of the observable $\gamma$-ray emission originates in leptonic processes and the accompanying hadronic processes contribute only to the higher energy part of the spectrum, only young PWNe (with the age $<1-3 \times 10^{3} \mathrm{yr}$ and at the typical distance of $2 \mathrm{kpc}$ ) should be detected by a $1 \mathrm{~km}^{2}$ neutrino detector with a rate above 1 event per year, i.e. above the atmospheric neutrino background within $1^{\circ}$ of the source. Between all considered PWNe observed in the $\mathrm{TeV} \gamma$-rays and containing a young $\gamma$-ray pulsar, or considered as likely objects for $\mathrm{TeV} \gamma$-ray detection, only neutrinos from the Crab Nebula should be observed by the $1 \mathrm{~km}^{2}$ with an event rate of 1 per yr. The neutrino event rate from the Vela SNR is comparable to that of the Crab nebula, but due to significantly lower energies of neutrinos and a much larger solid angle of the source on the sky, the signal from this object may not be extracted from the atmospheric neutrino background. The neutrino spectra from the other considered nebulae (pulsars) G 343.1-2.3 (PSR 1706-44), MSH15-52 (PSR 1509-58), 3C 58 (PSR J0205+6449), and CTB80 (PSR 1951+32), are significantly below the ANB, and the expected neutrino event rate is far below the sensitivity of a $1 \mathrm{~km}^{2}$ neutrino detector in a reasonable time of observation.

The neutrino rates, calculated in terms of the model for the high energy processes in PWNe considered here, are lower than those obtained by Guetta \& Amato (2002), who rescaled the observed $\gamma$-ray fluxes above $2 \mathrm{TeV}$ to the neutrino fluxes by simply assuming that all this $\gamma$-ray emission originates in hadronic processes. This assumption is in contradiction to the widely accepted leptonic model for the origin of the bulk emission from the WPNe. According to Guetta \& Amato (2002), Crab, Vela, PSR1706-44, and PSR1509-58 should be 
easily detected by the $1 \mathrm{~km}^{2}$ neutrino detector during one year. We have calculated the ratio of the neutrino luminosity to the $\gamma$-ray luminosity above $1 \mathrm{TeV}$ for the PWNe with reported $\mathrm{TeV} \gamma$-ray emission. This ratio is equal to $L_{\gamma} / L_{\gamma} \approx 58 \%$ (the Crab Nebula), 22\% (the Vela Nebula), and $0.5 \%$ (the nebula around PSR1707-44). Only in the case of the Crab Nebula should the predicted neutrino luminosity be close to the observed $\mathrm{TeV} \gamma$-ray luminosity above $1 \mathrm{TeV}$. The neutrino fluxes can be comparable to the observed $\gamma$-ray fluxes even for relatively old nebulae if high density medium is present close to the PWNa. As an example, in Bednarek \& Bartosik (2003) we consider the case of MSH15-52 (PSR 1509-58) which is close to high density medium (Seward et al. 1983). For this PWNa the ratio $L_{\nu} / L_{\gamma}$ is $\sim 77 \%$.

For all the considered nebulae we assumed that the pulsars are born with initial periods of $15 \mathrm{~ms}$, as derived for the Crab pulsar. In fact, pulsars with shorter periods can also explain the main observed parameters of these nebulae. If the initial periods of these pulsars are significantly shorter, the expected neutrino event rate increases. As an example, in Fig. 2 we consider the case of the pulsar with a period of $5 \mathrm{~ms}$ which produces the neutrino event rate by a factor of 2-3 higher than predicted for the $15 \mathrm{~ms}$ pulsar, for nebulae older than $\sim 10^{3} \mathrm{yr}$. Therefore, significant reduction of the initial period of the pulsar does not influence the main conclusions of this paper concerning the detection of neutrinos from specific nebulae by a $1 \mathrm{~km}^{2}$ detector.

We assumed that the density of the medium in which the supernova exploded is $0.3 \mathrm{~cm}^{-3}$. The pulsars surrounded by young supernova remnants, with similar parameters to the ones considered here, should produce much higher fluxes of neutrinos if they exploded in high density medium. For example, the presence of cloud with an average density of 300 particles $\mathrm{cm}^{-3}$ close to MSH15-52 (PSR 1509-58) should allow detection of neutrinos and explain the marginal detection of $\mathrm{TeV} \gamma$-rays from this object (Sako et al. 2000), as a result of radiation produced during interactions of hadrons with matter.

Acknowledgements. This work is supported by the Polish KBN grants No. 5P03D 02521 and PBZ-KBN-054/P03/2001.

\section{References}

Aharonian, F. A., \& Atoyan, A. M. 1995, Astropart. Phys., 3, 275 Aharonian, F. A., Atoyan, A. M., \& Kifune, T. 1997, MNRAS, 291, 162

Amato, E., Guetta, D., \& Blasi, P. 2003, A\&A, 402, 827

Arons, J. 1998, in Proc. Elba Conf. on Pulsars and their Nebulae, Mem. Soc. Ast. Ital., 69, 989

Atoyan, A. M., \& Aharonian, F. A. 1996, MNRAS, 278, 525

Beall, J. H., \& Bednarek, W. 2002, ApJ, 569, 343
Bednarek, W., \& Bartosik, M. 2003, A\&A, 405, 689 (BB03)

Bednarek, W., \& Protheroe, R. J. 1997, PRL, 79, 2616

Bednarek, W., \& Protheroe, R. J. 2002, Astropart. Phys., 16, 397

Berezinsky, V. S., \& Prilutsky, O. F. 1978, A\&A, 66, 325

Bietenholz, M. F., Kassim, N. E., \& Weiler, K. W. 2001, ApJ, 560, 772

Caraveo, P. A., De Luca, A., Mignani, R. P., \& Bignami, G. F. 2001, ApJ, 561, 930

Caswell, J. L., Murray, J. D., Roger, R. S., Cole, D. J., \& Cooke, D. J. 1975, A\&A, 45, 239

Cha, A., Sembach, K. M., \& Danks, A. C. 1999, ApJ, 515, L25

Chadwick, P. M., Dickinson, M. R., Dipper, N. A., et al. 1998, Astropart. Phys., 9, 131

Cheng, K. S., Cheung, T., Lau, M. M., Yu, K. N., \& Kwok, W. M. 1990, J. Phys. G, 16, 1115

Davidson, K., \& Fesen, R. A. 1985, ARA\&A, 23, 119

De Jager, O. C., \& Harding, A. K. 1992, ApJ, 396, 161

De Jager, O. C., Harding, A. K., Michelson, P. F., et al. 1996a, ApJ, 457, 253

De Jager, O. C., Harding, A. K., \& Strickman, M. S. 1996b, ApJ, 460, 729

Dermer, C. D., \& Atoyan, A. 2003, PRL, in press [astro-ph/0301030]

Du Plessis, De Jager, O. C., Buchner, S., et al. 1995, ApJ, 453, 746

Fesen, R. A. 1983, ApJ, 270, L53

Gaisser, T. K., \& Grillo, A. F. 1987, Phys. Rev. D, 39, 1481

Gallant, Y. A., \& Arons, J. 1994, ApJ, 435, 230

Gandhi, R. 2000, Nucl. Phys. Suppl., 91, 453

Guetta, D., \& Amato, E. 2003, Astropart. Phys., 19, 403

Guetta, D., \& Granot, J. 2003, PRL, 90, 191102

Hillas, A. M., Akerlof, C. W., Biller, S. D., et al. 1998, ApJ, 503, 744

Hoshino, M., Arons, J., Gallant, Y. A., \& Langdon, B. 1992, ApJ, 390, 454

Kifune, T., Tanimori, T., Ogio, S., et al. 1995, ApJ, 438, L91

Koo, B., Reach, W. T., Heiles, C., Fesen, R. A., \& Shull, J. M. 1990, ApJ, 346, 178

Lipari, P. 1993, Astropart. Phys., 1, 195

Murray, S. S., Slane, P. O., Seward, F. D., Ranson, S. M., \& Gaensler, B. M. 2002, ApJ, 568, 226

Ostriker, J. P., \& Gunn, J. E. 1971, ApJ, 164, L95

Protheroe, R. J., Bednarek, W., \& Luo, Q. 1998, Astropart. Phys., 9, 1

Razzaque, S., Meszaros, P., \& Waxman, E. 2003, PRL, in press [astro-ph/0212536]

Rees, M. J., \& Gunn, J. E. 1974, MNRAS, 167, 1

Roberts, D. A., Goss, W. M., Kalberla, P. M. W., Herbstmeier, U., \& Schwarz, U. J. 1993, A\&A, 274, 427

Sako, T., Matsubara, Y., Muraki, Y., et al. 2000, ApJ, 537, 422

Seward, F. D., Harnden, F. R. Jr., Murdin, P., \& Clark, D. H. 1983, ApJ, 267, 698

Strom, R. G., Angerhofer, P. E., \& Velusamy, T. 1980, Nature, 284, 38

Taylor, A. P., \& Cordes, J. M. 1993, ApJ, 411, 674

Thorsett, S. E. 1992, Nature, 356, 690

Vietri, M., \& Stella, L. 1998, ApJ, 507, L45

Wang, Z. R., \& Seward, F. D. 1984, ApJ, 285, 607

Wdowczyk, J., \& Wolfendale, A. W. 1987, J. Phys. G, 13, 411 